Die AGQ-FMH erstellt in Zusammenarbeit mit der Abteilung DDQ Grundlagenpapiere zu verschiedenen Themen im Bereich Qualität, die in verkürzter Form in der Schweizerischen Ärztezeitung veröffentlicht werden. Die ausführliche Version der Grundlagenpapiere können Mitglieder bei der FMH anfordern (ddq@fmh.ch). Die FMH nimmt auf der Basis der erarbeiteten Grundlagen Stellung zum Thema und gelangt über das Papier «Die Meinung der FMH» mit ihrer Position an die Öffentlichkeit.

\title{
Qualitätsmanagement und Zertifizierung der ärztlichen Leistungserbringung: Aufwand und Nutzen
}

\author{
Arbeitsgruppe Qualität der FMH \\ $(A G Q-F M H)$ *
}

\footnotetext{
* Mitglieder AGQ-FMH (alphabetische Reihenfolge): Patrick Bovier, Martina Hersperger, Olivier Kappeler, Esther Kraft, Francesca Mainieri, Claude Oppikofer, Michael Peltenburg, Jean-Claude Roches, Iris Stucki, Hans Anton Vogel, Georg von Below.
}

\begin{abstract}
Einleitung
Das Schaffen einer hohen Versorgungsqualität, das heisst den Patienten zur richtigen Zeit am richtigen Ort mit der bestmöglichen medizinischen Leistung zu versorgen, ist der Leitgedanke der ärztlichen Berufsausübung. Entsprechend zählt die Qualitätsförderung zum Selbstverständnis der Ärztin/des Arztes und ist in der Standesordnung der FMH verankert.

Qualitätsmanagement der ärztlichen Leistungserbringung kann über eine Bandbreite von Massnahmen erfolgen: Es gibt international und national anerkannte QM-Systeme, Indikatorensets für einzelne Fachbereiche, standardisierte Instrumente der Qualitätssicherung. Es können aber auch individualisierte Systeme in einzelnen Praxen zur Anwendung kommen. Das Ziel ist stets die systematische kontinuierliche Verbesserung der Qualität.
\end{abstract}

\section{Juristische Grundlagen zur Qualitätssicherung in der Medizin}

Art. 58 Abs. 1 KVG sieht vor, dass der Bundesrat nach Anhören der interessierten Organisationen systematische wissenschaftliche Kontrollen zur Sicherung der Qualität oder des zweckmässigen Einsatzes der von der obligatorischen Krankenpflegeversicherung übernommenen Leistungen vorsehen kann. Mit Art. 77 Abs. 1 KVV hat der Bundesrat die Aufgabe der Qualitätssicherung den Leistungserbringern und deren Verbänden übertragen. In den Rahmenverträgen von $\mathrm{H}+$ und der FMH mit der santésuisse (TARMED) ist die Qualitätssicherung allgemein geregelt: Die Spitäler resp. Ärzte verpflichten sich zur Mitwirkung bei Massnahmen für Sicherung und Kontrolle der Qualität nach Art. 58 KVG. Konkrete Massnahmen wurden jedoch noch nicht defi- niert. Es gibt einzelne spezifische Bereiche mit expliziten Qualitätssicherungsverträgen, beispielsweise für das Labor (Qualab) oder für die Spartenanerkennung von Operationssälen gemäss TARMED. Über die kantonalen Leistungsverträge sind Bestimmungen zur Qualitätssicherung zusätzlich geregelt.

In der UV-, MV- und IV-Gesetzgebung gibt es keine expliziten Grundlagen zur Qualitätssicherung. In der obligatorischen Unfallversicherung wird das Wort «Qualität» nicht erwähnt [1].

Die Geschäftsprüfungskommission des Ständerates stellt im Schreiben an den Bundesrat vom 13. November 2007 fest, «dass im Bereich der Qualitätssicherung noch beträchtliches Optimierungspotenzial besteht und der Bund seine gesetzlichen Kompetenzen nicht konsequent genug nutzt». Sie fordert deshalb eine klare und verbindliche Strategie, welche Massnahmen, Verantwortlichkeiten und Fristen für die Umsetzung des Qualitätssicherungsauftrags des Bundes definiert.

\section{Begriffe Qualität, Qualitätsmanagement, Qualitätsmanagementsysteme}

Es gibt zahlreiche Versuche, Qualität zu definieren, so z.B. die weitverbreitete Definition der ISO-Norm, die unter Qualität «das Vermögen einer Gesamtheit inhärenter Merkmale eines Produktes (Dienstleistung), Systems oder Prozesses zur Erfüllung von Forderungen von Kunden und anderen interessierten Parteien» versteht (DIN EN ISO 9000:2000). Auf die medizinische Leistungserstellung bezogen kann Qualität folgendermassen definiert werden: «Quality of care is the degree to which health services for individuals and populations increase the likelihood of desired health outcomes and are consistent 
with current professional knowledge» [2]. Campbell ergänzt das systemorienterte Qualitätskonzept von Donebedian (Struktur, Prozess, Ergebnis) [3] um die Dimensionen Zugang und Effizienz [4]. Im Leitbild ärztliche Qualität der FMH wird bei der Beschreibung der Qualität der ärztlichen Leistungserbringung betont, dass Qualität als eine dynamische, veränderbare Grösse Teil eines lernenden Systems und somit der kontinuierlichen Verbesserung unterworfen ist sowie als Koproduktion mit den Anspruchsgruppen ausgehandelt und umgesetzt werden muss [5].

Der Begriff Qualitätsmanagement kann definiert werden als «aufeinander abgestimmte Tätigkeiten zur Leitung und Lenkung einer Organisation bezüglich Qualität» (DIN EN ISO 9000). Nach EN ISO 9000 ist «Qualitätssicherung» «Teil des Qualitätsmanagements, der auf das Erzeugen von Vertrauen darauf gerichtet ist, dass Qualitätsanforderungen erfüllt werden». Die Qualitätssicherung sorgt demnach für die Einhaltung der vom Qualitätsmanagement festgelegten Massnahmen.

Unter einem Qualitätsmanagementsystem wird die Systematik der Struktur-, Planungs- und Handlungsvorgaben zur Erfüllung der Aufgaben des Qualitätsmanagements verstanden [6]. Grundlage des systematischen Qualitätsmanagements ist der Plan-Do-Check-Act-Zyklus (PDCAZyklus oder auch Deming-Zyklus genannt). Im Zentrum steht das Prinzip der kontinuierlichen Verbesserung. Dieser Verbesserungsprozess ist heute in allen gängigen QM-Systemen integriert. Der Europarat definiert ein Qualitätsmanagementsystem im Gesundheitsbereich als «set of integrated and planned acitivities and measures at various levels in the health care organisation, aimed at continously assuring and improving the quality of patient care». So verstanden ist das Qualitätsmanagementsystem nichts, was der ärztlichen Tätigkeit fremd ist, sondern eine originär ärztliche Aufgabe. Was bisher weniger verankert ist, ist eine durchgehende Methodik [6]. Die kontinuierliche Qualitätsverbesserung (Continuous Quality Improvement) schliesslich umfasst die Gesamtheit aller Tätigkeiten, welche zum Ziel haben, die Qualität ständig zu verbessern.

a Richtlinie des Gemeinsamen Bundesausschusses (2006) über grundsätzliche Anforderungen an ein einrichtungsinternes Qualitätsmanagement für die an der vertragsärztlichen Versorgung teil nehmenden Ärzte, Psychotherapeuten und medizinischen Versorgungszentren. mentsystem wird die Bewertung anders und unter anderem Namen vorgenommen [7]. Neben der Zertifizierung anhand der DIN EN ISO oder den «Akkreditierungsverfahren» nach Vorbild der USA (JCAHO) kommen auch spezifisch europäische Verfahren wie die Vergabe der Europäischen Qualitätsauszeichnungen (EFQM) durch (Self-)Assessments oder adaptierte Verfahren (EPA) zur Anwendung. In der Schweiz wurden spezifisch QMS für Spitäler (sanaCERT), für Praxen (EPA und EQUAM) und für Ärztenetzwerke (Quali-med-net) entwickelt. Im Anhang I findet sich ein Überblick über einige wichtige Qualitätsmanagementsysteme, welche derzeit international und lokal im stationären und/oder ambulanten Sektor Anwendung finden.

Arztpraxen und Spitäler in der Schweiz können freiwillig Qualitätsmanagementsysteme einführen und zertifizieren. Im Gegensatz dazu sind in Deutschland die Vertragsärzte gesetzlich verpflichtet, einrichtungsintern ein Qualitätsmanagement einzuführen und weiterzuentwickeln. ${ }^{\mathrm{a}}$

Zur Implementierung und Zertifizierung von Qualitätsmanagementsystemen in der Schweiz sind folgende Angaben vorhanden:

- Im stationären Bereich wenden gemäss H+ 85\% der Betriebe mindestens ein Qualitätsmanagementsystem für den ganzen Betrieb oder einzelne Kliniken an [8].

- Von EPA sind die folgenden Zahlen bekannt: Seit 2005 haben zirka 130 Praxen das EPAAssessment durchlaufen, aktuell läuft EPA in 31 Praxen. 30210 Patienten haben ihren Arzt beurteilt (Stand 21. April 2008).

- 240 Ärzte verfügen über ein EQUAM-Zertifikat. Sie sind in 6 Netzen und 6 HMO-Praxen organisiert. Von den 240 Ärzten sind 15 nicht in eine Managed-Care-Organisation eingebunden (Stand 21. April 2008).

- ÄNW: 38 Mitglieder, detaillierte Angaben über den Grad der Implementierung von Quali-med-net sind nicht vorhanden (Stand 28. August 2007)

- Von ISO sind keine spezifischen Daten in bezug auf Spitäler und Praxen erhältlich, die auf der Website publizierten Zertifizierungen im Gesundheitsbereich beinhalten auch Altersheime, Pflegeeinrichtungen usw.

- sanaCERT hat seit 200315 Akutspitäler zertifiziert (Stand 21. April 2008).

Abschliessend kann nicht davon gesprochen werden, dass sich in der Schweiz ein bestimmtes Qualitätsmanagementsystem im ambulanten oder stationären Sektor etabliert hat. 


\section{Qualitätsentwicklung und ärztliches Handeln: Aufwand und Nutzen}

Die Einführung und Aufrechterhaltung eines Qualitätsmanagementsystems ist mit Aufwand verbunden - diesem Aufwand steht sinnvollerweise ein Nutzen gegenüber. Die Höhe des Aufwandes hängt vom gewählten System ab. Entsprechend ist auch die Akquisition eines QMZertifikates mit Kosten verbunden [9]. Unseres Wissens wurden bisher weder der Nutzen der Qualitätsmanagementsysteme noch die Zertifizierung auf die Qualität der ärztlichen Leistungserbringung systematisch untersucht und erwiesen. Als Nutzen von Zertifikaten wird von gewissen Autoren die wettbewerbliche Bedeutung genannt: Gegenüber dem nichtzertifizierten Konkurrenten entsteht der subjektive Eindruck, dass die Leistung des zertifizierten Unternehmens von höherer Qualität sei [9].

\section{Abbildung 1}

Qualitätsentwicklung: Vom Aufwand zum Nutzen (Quelle: AGQ-FMH).

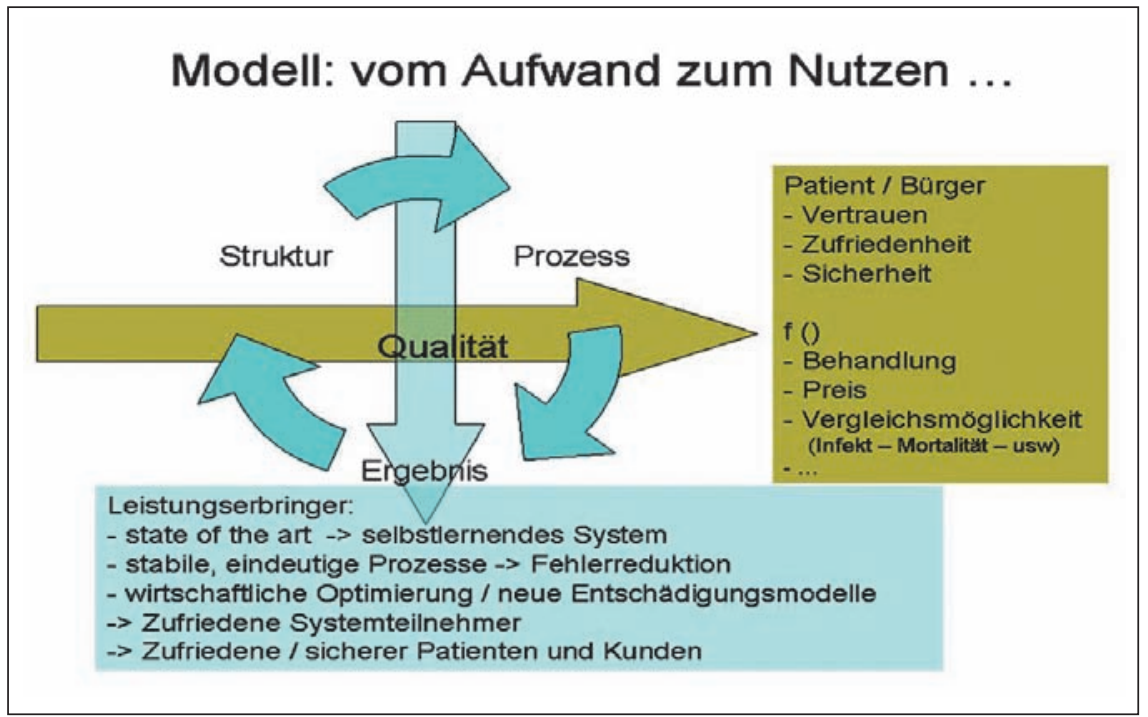

b Stärke von Qualitätsindikatoren nach Marshall in Schneider et al [11]: z. B. über Transparenz Vertrauen fördern.
Für die ärztliche Berufspraxis spielen bei der Bewertung des Nutzens auch die immateriellen Aspekte eine zentrale Rolle, und zwar sowohl auf der Ebene des Arztes (Beteiligte) als auch auf der Ebene der Patienten (Betroffene). Ein Nutzen eines Qualitätsmanagementsystems kann ein Betrieb vermutlich aus der Aufbauphase des QMSystems ziehen, indem Arbeits- und Prozessabläufe analysiert und gegebenenfalls verbessert werden. Die AGQ geht davon aus, dass ein Qualitätsmanagementsystem durch die Transparenz der Qualität gegenüber den Patienten und der Bevölkerung Rechenschaft über das Handeln des Arztes ablegt, wodurch das Vertrauen in die ärzt- liche Behandlung erhöht werden kann. ${ }^{\mathrm{b}}$ Durch die Optimierung der Abläufe, auch spezifisch auf Patienten bezogen (z. B. kürzere Wartezeiten durch effizientere Praxisorganisation), kann schliesslich die subjektive Patientenzufriedenheit im Sinne der Servicequalität positiv beeinflusst werden.

Die Abbildung 1 verdeutlicht den Zusammenhang zwischen Aufwand und Nutzen für den einzelnen Arzt. Es gibt zwei Ebenen des Nutzens, auf der einen Seite steht der Patient/Bürger, auf der anderen Seite der Leistungserbringer. Der Arzt ist interessiert an stabilen, sicheren Abläufen, damit die Fehlerquellen gering sind und wenige Komplikationen auftreten. Der Patient möchte sicher sein, vertrauen können, er möchte informiert entscheiden und auswählen können:

Eine randomisierte Kontrollstudie in den Niederlanden zeigte, dass allein durch Patientenbefragungen die Patientenzufriedenheit nicht signifikant verbessert werden konnte, auch nicht für Bereiche, in denen Ärzte explizit Massnahmen zur Verbesserung unternahmen. Die Ärzte selbst jedoch berichteten über Veränderungen in ihrem Verhalten und den organisatorischen Abläufen [10]. Verschiedene Autoren weisen darauf hin, dass vereinzelte Massnahmen keine Veränderung bewirken, sondern dass ein multimodaler Ansatz zur Anwendung kommen sollte [10-12]. Ebenso müssen mögliche Barrieren vorgängig identifiziert und die Massnahmen entsprechend abgestimmt werden [12].

\section{Individuelles ärztliches Handeln}

Massnahmen zur Qualitätsentwicklung können auch für das ärztliche Handeln im Alltag individualisiert werden. Bei individuellen Massnahmen ist man jedoch mit dem Problem der fehlenden Vergleichbarkeit konfrontiert.

Aus der Sicht des einzelnen Arztes steht die Sicherstellung, Verbesserung und Optimierung des ärztlichen Handelns im Zentrum. Dabei muss der Arzt verschiedenen Anspruchsgruppen gerecht werden (Arztkollegen, Mitarbeitende, Patienten, Versicherer). Die Grundlage der Qualitätsentwicklung ist die systematische, kontinuierliche Verbesserung aller Leistungen. Damit sichergestellt werden kann, dass die geplanten Verbesserungsmassnahmen zur Qualitätsentwicklung beitragen, müssen folgende Kriterien umgesetzt werden:

Habe ich als Arzt ...

1. ... messbare Ziele in den Bereichen Struktur, Prozess und Ergebnis definiert?

2. ... nachweisbare Indikatoren daraus abgeleitet? 
3. ... Kontrollmechanismen integriert, anhand deren ich periodisch die Indikatoren überprüfen kann (Soll-Ist-Vergleich)?

4. ... Massnahmen zur Verbesserung eingeleitet, die die Effizienz und die Effektivität steigern?

5. ... Instrumente zum Risiko-, Fehler- und Beschwerdemanagement eingeführt?

6. ... meine Resultate dokumentiert und transparent gemacht?

7. ... aus den Resultaten Konsequenzen gezogen?

Die sieben Kriterien sollen verdeutlichen, dass ein Kreislauf entsteht, bei dem aus den Resultaten von Verbesserungsmassnahmen Konsequenzen gezogen werden können, die wieder $\mathrm{zu}$ neuen Zielen führen. Im folgenden wird eine Möglichkeit zur Qualitätsentwicklung in einer konsiliarisch tätigen Facharztpraxis erläutert (Tab. 1).

\section{Literatur}

1 Frésard J-M. L'assurance-accidents obligatoire. In: Schweizerisches Bundesverwaltungsrecht (SVBR). Band XIV. Basel: Helbling \& Lichtenhahn; 2007. S. 825-1040.

2 Lohr KN, Schroeder SA. A strategy for quality assurance in Medicare. N Engl J Med. 1990;322:707-12.

3 Donabedian A. The seven pillars of quality. Arch Path Lab Med. 1990;114:1115-8.

4 Campbell SM, Roland MO, Buetow SA. Defining quality of care. Soc Sci Med. 2000;51(11):1611-25.

5 Peltenburg M, Kernen H, Schneider P, von Below GC, Waldis G, et al. Qualität - ein Zusammenspiel aller Kräfte im ärztlichen Umfeld. Schweiz Ärztezeitung. 2005;86(18):1055-8.

6 Nüllen H, Noppeney T. Lehrbuch Qualitätsmanagement in der Arztpraxis. Entwicklung und Einführung eines QMS. Köln: Deutscher Ärzte-Verlag; 2007.

7 Ziegler M. I-1.1.1. Zertifizierung, Assessment, Akkreditierung - Qualitätsmanagementmodelle und ihre Terminologie. In: Scheibe, O, Ekkernkamp A (Hrsg.). Qualitätsmanagement in der Medizin. Handbuch für Klinik und Praxis. Landsberg am Lech: ecomed; 1996.
8 Favre C. H+ qualité: Neues Label für Patientensicherheit und Qualität in Spitälern und Kliniken. Präsentation an der Medienkonferenz zum Nationalen Spitaltag vom 8. September 2007 (23. August 2007).

9 Ollenschläger G. Gedanken zur Zertifizierung in der ambulanten Versorgung. ZaeFQ. 2000;94:645-9.

10 Vingerhoets M, Wensing M, Grol R. Feedback of patients' evaluations of general practice care: a randomised trial. Qual Health Care. 2001;10(4):224-8.

11 Schneider A, Broge B, Szecsenyi J. Müssen wir messen, um (noch) besser werden zu können? Die Bedeutung von Qualitätsindikatoren in strukturierten Behandlungsprogrammen und Qualitätsmanagement. Z Allg Med. 2003;79:547-52.

12 Wensing M, Van der Weijden T, Grol R. Implementing guidelines and innovations in general practice: which interventions are effective? Br J Gen Pract. 1998;48:991-7.

13 Hennig S, Greiner W. Qualitätsmanagementsysteme in der ambulanten ärztlichen Versorgung Ein Vergleich. Gesundheitsökonomie und Qualitätsmanagement. 2007;12:235-46.

14 Denz C, Krieter H, von Ackern K. Stellenwert des Qualitätsmanagements und der Zertifizierung im Krankenhausbereich. Gesundheitsökonomie und Qualitätsmanagement. 2004;6:382-91.

15 Donahue KT, van Ostenberg P. Joint Commission International accreditation: relationship to four models of evaluation. Int J Qual Health Care. 2000;12:243-6.

16 Klingenberger D. Health Maintenance Organizations in der Schweiz. IDZ-Information 1/2002.

17 Europäisches Praxisassessment (EPA). Die lernende Praxis: Qualitätsmanagement durch Assessment und Weiterentwicklung.

18 Ziegler M. I-1.2. DIN EN ISO 9000 bis 9004. In: Scheibe, O, Ekkernkamp A (Hrsg.). Qualitätsmanagement in der Medizin. Handbuch für Klinik und Praxis. Landsberg am Lech: ecomed; 1996.

19 Ziegler M. I-1.3. Das Modell der Euorpean Foundation for Quality Management (EFQM). In: Scheibe O, Ekkernkamp A (Hrsg.). Qualitätsmanagement in der Medizin. Handbuch für Klinik und Praxis. Landsberg am Lech: ecomed; 1996.

20 Ziegler M. I-1.5. Joint Commission on Accreditation of Healthcare Organizations (JCAHO). In: Scheibe, O, Ekkernkamp A (Hrsg.). Qualitätsmanagement in der Medizin. Handbuch für Klinik und Praxis. Landsberg am Lech: ecomed; 1996.

21 Christen P. Quali-med-net: Das Instrument zur Qualitätsbeurteilung von Ärztenetzwerken. Powerpoint-Präsentation im Rahmen der Steuergruppe AGQ-FMH (28. August 2007). 


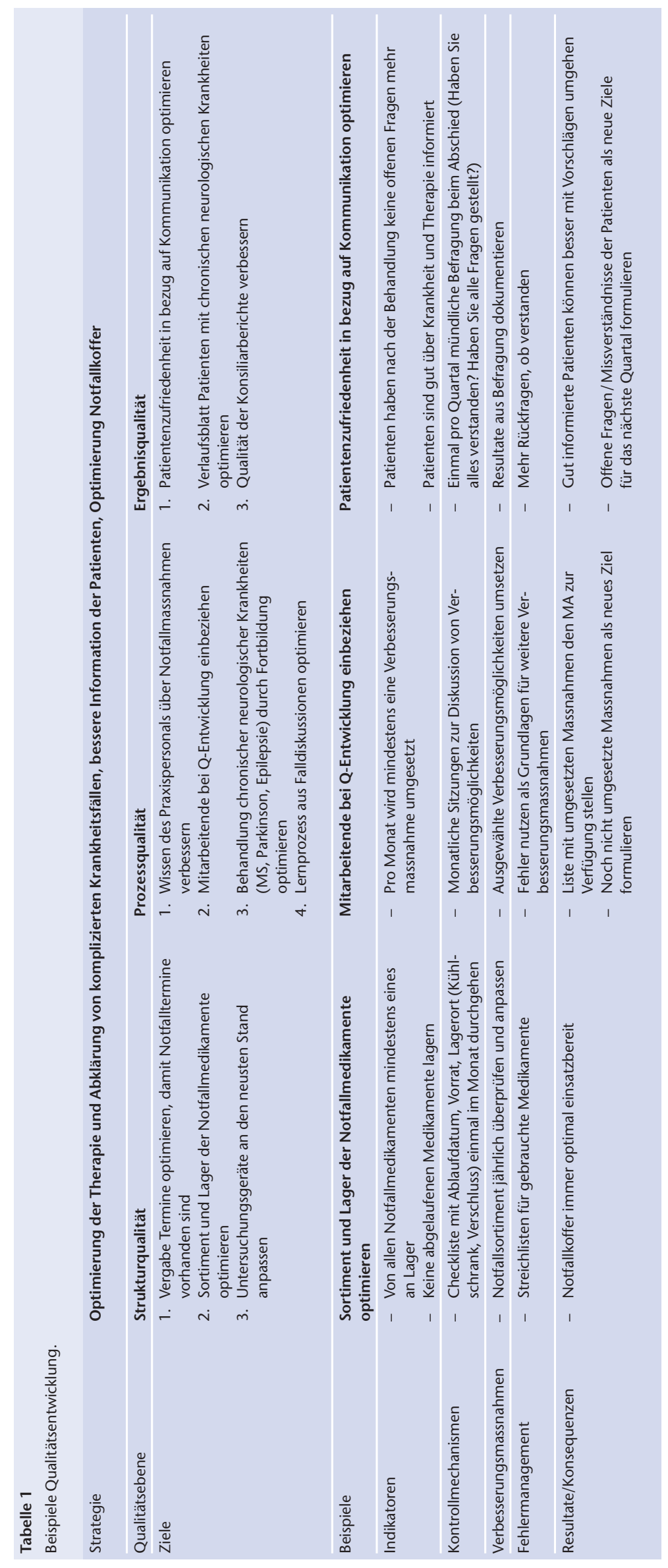




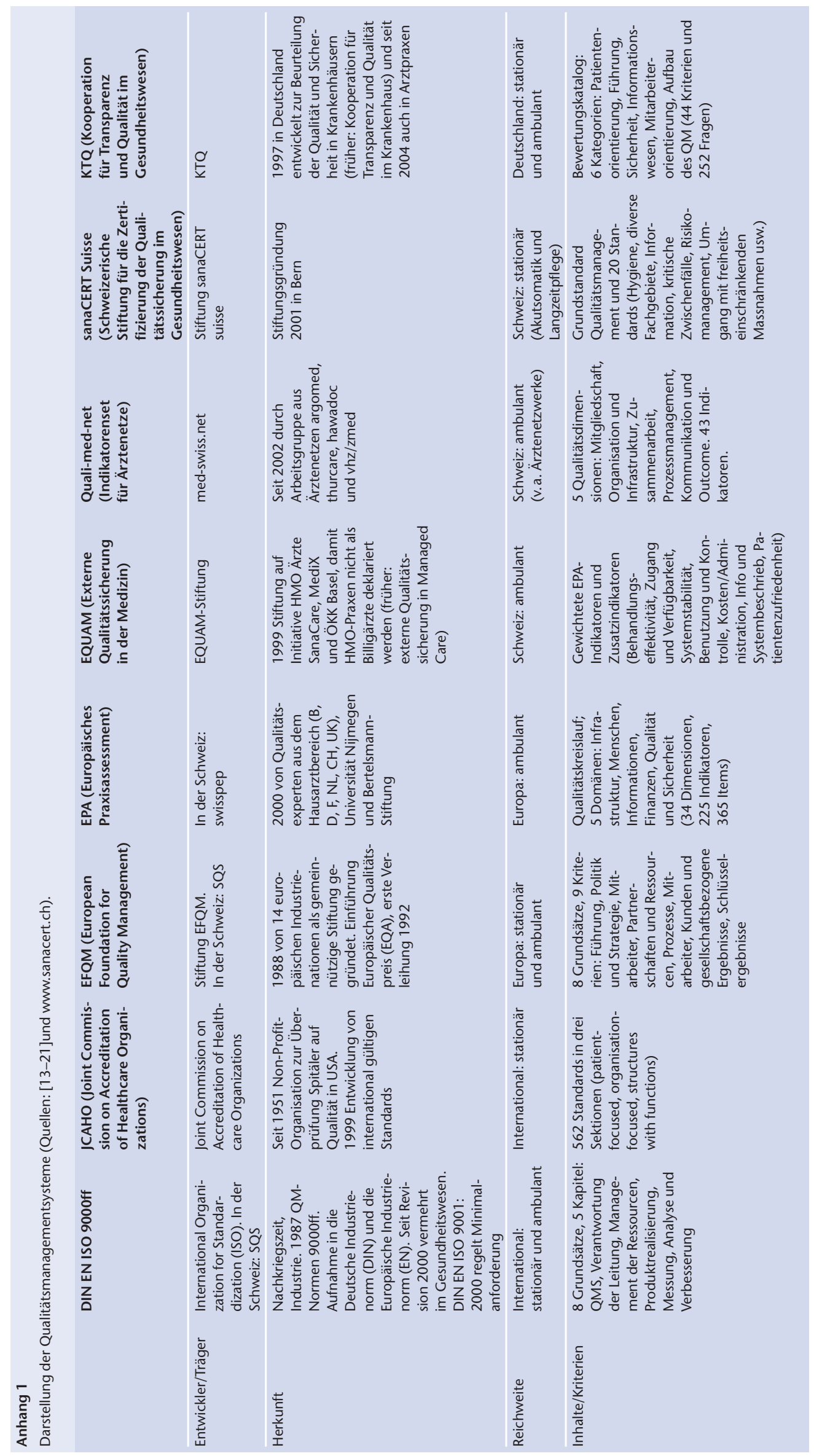




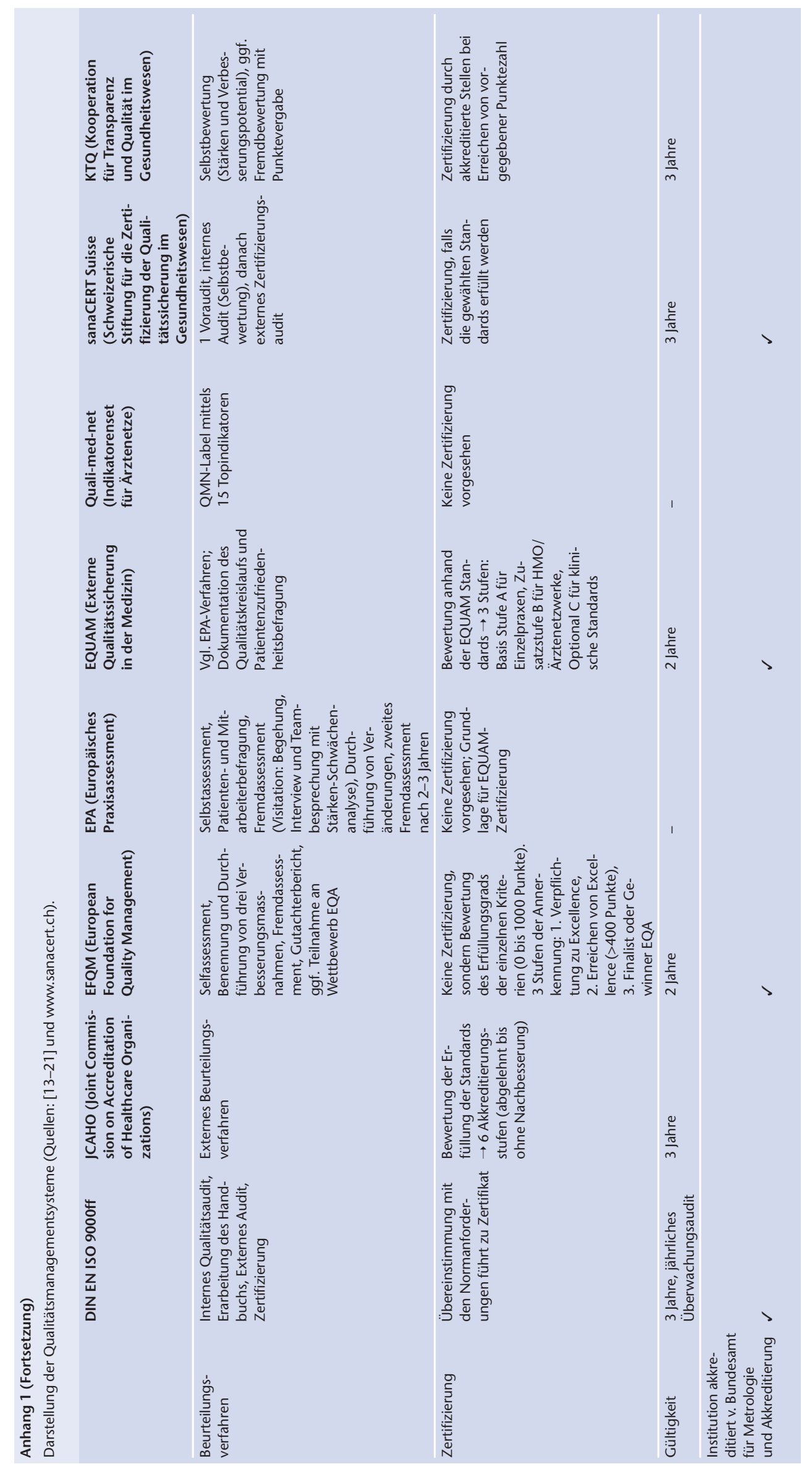

